\title{
Diagnostic utility of neural stem and progenitor cell markers nestin and SOX2 in distinguishing nodal melanocytic nevi from metastatic melanomas
}

\author{
Pei-Ling Chen ${ }^{1}$, Wei-Shen Chen ${ }^{1}$, Jianping $\mathrm{Li}^{1}$, Anne C Lind ${ }^{1}$ and Dongsi $\mathrm{Lu}^{1,2}$ \\ ${ }^{1}$ Department of Pathology and Immunology, Washington University School of Medicine, St Louis, MO, USA \\ and ${ }^{2}$ Department of Pathology, St Luke's Hospital, Chesterfield, MO, USA
}

\begin{abstract}
Sentinel lymph node evaluation is a critical component of melanoma staging, and lymph node status provides one of the most powerful predictors of melanoma recurrence and survival. One of the well-known diagnostic pitfalls in melanoma sentinel lymph node evaluation is the presence of nodal melanocytic nevi, which has been demonstrated in up to $26 \%$ of lymphadenectomy specimens and specifically in melanoma patients. Melanocytic markers enhance the sensitivity of melanoma detection in sentinel lymph nodes. However, established markers such as anti-melan-A/MART1, S100 protein and SOX10 antibodies cannot discriminate melanoma metastasis from nodal nevi. Recent studies have demonstrated strong expression of neural stem/progenitor cell markers nestin and SOX2 in melanoma. In this study, we tested the diagnostic utility of nestin and SOX2 in differentiating metastatic melanomas from nodal nevi. Twenty-three lymph nodes with metastatic melanomas and 17 with nodal nevi were examined. Of the 23 metastatic melanomas, 18 showed diffuse and strong $(3+)$ nestin, 4 showed rare cells with strong $(3+)$ nestin, and one showed diffuse but faint $(1+)$ nestin staining. Nuclear SOX2 was positive in 13 metastatic melanomas. In contrast, 15 nodal nevi showed no nestin, and 2 showed rare cells with very faint $(<1+)$ nestin staining. SOX2 was negative in 13 nodal nevi. Overall, nestin was strongly expressed in metastatic melanomas ( $n=22 / 23 ; 96 \%)$, but not in nodal melanocytic nevi $(n=15 / 17 ; 88 \%$; $P<0.0001)$. SOX2 was also expressed in metastatic melanomas $(n=13 / 23 ; 57 \%)$ but not in the majority of nodal melanocytic nevi $(n=13 / 16 ; 81 \% ; P=0.02)$. In one lymph node harboring metastatic melan-A-negative desmoplastic melanoma, nestin and SOX2 strongly highlighted the infiltrating tumor cells, suggesting the potential clinical value of these two markers in desmoplastic melanoma lymph node biopsies. This study provides evidence that nestin and SOX2 can effectively differentiate nodal melanocytic nevi from metastatic melanomas and serve as powerful diagnostic adjuncts in melanoma staging.
\end{abstract}

Modern Pathology (2013) 26, 44-53; doi:10.1038/modpathol.2012.132; published online 17 August 2012

Keywords: metastatic melanomas; nestin; nodal melanocytic nevi; SOX2

Malignant melanoma is a malignant tumor of melanocytes that accounts for less than $10 \%$ of all skin cancer diagnoses but the majority of skin cancerrelated deaths. ${ }^{1}$ For patients diagnosed with malignant melanoma, sentinel lymph node biopsy is an essential component of tumor staging, and lymph node status provides one of the most powerful predictors of melanoma recurrence and survival. ${ }^{2-4}$ When melanoma metastasizes, a regional lymph node

Correspondence: D Lu, MD, PhD, Department of Pathology, St Luke's Hospital, Chesterfield, MO 63017, USA.

Email: dongsi.lu@stlukes-stl.com

Received 29 February 2012; revised 27 June 2012; accepted 28 June 2012; published online 17 August 2012 is the most common site, and tumor burden can range from rare tumor cells to complete effacement of lymph nodes. The presence of either micro- or macrometastasis in a single lymph node changes melanoma staging from stage I to stage III. ${ }^{5}$ In addition, a single positive lymph node signifies a decrease in 10-year survival from $95 \%$ (stage IA) to $68 \%$ (stage IIIA), and with more than three positive lymph nodes, 10-year survival rate drops to $24 \% .^{5}$ Although controversial, many surgeons perform completion lymphadenectomy based on the histological diagnosis of a single positive sentinel lymph node, ${ }^{2}$ and high-dose interferon- $\alpha 2 b$ is offered as an adjuvant treatment option for stage III melanoma. As sentinel lymph node evaluation carries significant prognostic 
and therapeutic implications, accurate assessment of sentinel lymph node biopsies is crucial for appropriate tumor staging and patient management.

The recently published seventh edition of the AJCC staging system for cutaneous melanoma has incorporated two important changes specific to the handling and interpretation of sentinel lymph node biopsies. ${ }^{5}$ First, unlike breast cancer, there is no lower threshold of tumor burden to define regional nodal metastasis, reflecting the view that even small tumor volume in lymph nodes may be clinically significant. ${ }^{6}$ Second, for the purpose of melanoma staging, nodal micrometastasis is now defined using either $\mathrm{H} \& \mathrm{E}$ or immunohistochemical staining, which must include at least one melanocyte-specific marker. These two changes in AJCC tumor staging herald more prevalent use of immunohistochemical markers for the detection of melanoma micrometastasis and an increased likelihood of encountering challenging nodal melanocytic lesions due to more sensitive detection methods.

One of the major diagnostic pitfalls in sentinel lymph node biopsies from melanoma patients is the presence of nodal melanocytic nevi. ${ }^{7,8}$ Benign melanocytic nevi are reportedly identified in $8-26 \%$ of lymphadenectomy specimens and occur more frequently in melanoma patients, specifically in sentinel lymph nodes. ${ }^{8}$ Histologically, nodal melanocytic nevi consist of aggregates of cytologically bland melanocytes that reside in the lymph node capsule and trabeculae, whereas metastatic melanomas are predominantly subcapsular, sinusoidal and intraparenchymal, and show cytological features of malignancy. ${ }^{9}$ Nonetheless, as melanomas often display a wide spectrum of cytological features and can be morphologically bland, as in nevoid melanoma, the distinction between benign nodal nevi and melanoma metastases can be difficult. To complicate the matter further, both metastatic melanomas and nodal melanocytic nevi can occupy unusual sites within the lymph nodes. It has been shown that melanoma cells can seed lymph node capsules, ${ }^{10}$ and nevoid aggregates have been identified in the subcapsular space and even within nodal parenchyma, ${ }^{7}$ mimicking metastasis. On the basis of morphological criteria alone, distinguishing nodal melanocytic nevi from nodal melanomas can be very difficult, especially when the melanocytic focus in question is small and lacks overt cytological atypia. In such histologically ambiguous lesions, care must be taken to avoid over-diagnosing nodal nevi as metastatic melanomas or under-diagnosing tumor deposits as nodal melanocytic nevi.

Many melanocytic markers are routinely used in the evaluation of microscopic metastasis, yet no single immunostain is adequately specific in differentiating melanoma metastases from nodal melanocytic nevi. ${ }^{11}$ Melan-A/MART1 and SOX10 are highly sensitive for melanoma. ${ }^{12,13}$ However, they indiscriminately highlight melanoma and nevus cells, and cannot distinguish between these two entities. ${ }^{13,14}$ HMB-45 is an antibody directed against glycoprotein gp100 in premelanosomes, yet it is also positive in isolated or aggregated melanophages in sentinel lymph nodes. ${ }^{15}$ S100 protein, one of the most sensitive markers for melanoma, is also expressed in nodal melanocytic nevi and diffusely highlights dendritic cells, ${ }^{14,16}$ making the interpretation of this marker particularly confusing in lymph node specimens.

With the recent isolation of tumorigenic stem cells in human, ${ }^{17}$ we postulated that even though metastatic melanomas and nodal melanocytic nevi share common lineage, melanomas are more likely to express neural stem/progenitor cell markers than nodal melanocytic nevi, which are presumably terminally differentiated cells. A few studies have demonstrated that the neural stem/progenitor cell marker nestin, a type VI intermediate filament transiently expressed in early migrating and proliferating neuroectodermal cells, ${ }^{18}$ is expressed in primary and metastatic melanomas. ${ }^{19-23}$ Furthermore, this expression seem to correlate with more advanced disease and decreased 5-year survival. ${ }^{24,25}$ In contrast, the majority of melanocytic nevi, even dysplastic nevi, showed no or weak nestin staining. ${ }^{20,23}$ SOX2, or SRY (sex determining region Y)-box 2, is a transcription factor expressed by neural progenitor cells throughout the vertebrate CNS, and can bind to and regulate the nestin core enhancer. ${ }^{26}$ It is also one of the key transcription factors essential for the induction of pluripotent stem cells from fibroblasts in mice. ${ }^{27}$ Recently, it has been shown on whole tissue sections that metastatic melanomas expressed both nestin and SOX2, but no cutaneous melanocytic nevi were positive for both markers. ${ }^{23}$

The aim of this study was to investigate the diagnostic utility of neural stem/progenitor cell markers nestin and SOX2 in differentiating nodal melanocytic nevi from metastatic melanomas.

\section{Materials and methods}

\section{Study Population and Case Selection}

This study was approved by the Institutional Review Board of Washington University in St Louis. The Department of Pathology database was searched for cases diagnosed with nodal melanocytic nevi from 1993 to 2011, and a matching number of metastatic melanomas to the lymph nodes were randomly selected for this study. Archival formalinfixed, paraffin-embedded tissue blocks and slides were retrieved from our surgical pathology files. All H\&E-stained slides were independently reviewed by three study pathologists (DL, AL and PLC). A total of 41 surgical specimens were included in this study: 23 lymph nodes with metastatic melanomas (16 sentinel, 7 non-sentinel), 17 lymph nodes with nodal melanocytic nevi (8 sentinel, 9 non-sentinel; 5 with history of melanoma) and 1 lymph node with 
'subcapsular/intranodal melanocytic rest.' Patient demographics (age, gender, primary diagnosis, specimen type and location) are detailed in Table 1.

\section{Immunohistochemistry}

Results of each immunostain were independently reviewed by three study pathologists (AL, DL and PLC). Immunostain for nestin (Milipore, 1:100, no. MAB5326) was performed on a Ventana Benchmark XT automated immunostainer (Ventana Medical Systems, Tucson, AZ, USA) according to standard protocols after heat-induced antigen retrieval.

Table 1 Patient demographics

Metastatic melanoma

\begin{tabular}{lcclc}
\hline No. & Sex & Age & Location & Sentinel \\
\hline 1 & $\mathrm{M}$ & 75 & Jugular & $\mathrm{Y}$ \\
2 & $\mathrm{~F}$ & 19 & Groin & $\mathrm{Y}$ \\
3 & $\mathrm{M}$ & 52 & Back & $\mathrm{Y}$ \\
4 & $\mathrm{M}$ & 72 & Axillary & $\mathrm{Y}$ \\
5 & $\mathrm{M}$ & 75 & Axillary & $\mathrm{Y}$ \\
6 & $\mathrm{M}$ & 63 & Axillary & $\mathrm{Y}$ \\
7 & $\mathrm{M}$ & 72 & Groin & $\mathrm{Y}$ \\
8 & $\mathrm{M}$ & 78 & Upper arm & $\mathrm{Y}$ \\
9 & $\mathrm{M}$ & 72 & Neck & $\mathrm{Y}$ \\
10 & $\mathrm{M}$ & 58 & Supraclavicular & $\mathrm{Y}$ \\
11 & $\mathrm{M}$ & 68 & Inguinal & $\mathrm{Y}$ \\
12 & $\mathrm{~F}$ & 39 & Groin & $\mathrm{Y}$ \\
13 & $\mathrm{M}$ & 29 & Axillary & $\mathrm{Y}$ \\
14 & $\mathrm{M}$ & 67 & Axillary & $\mathrm{N}$ \\
15 & $\mathrm{M}$ & 53 & Axillary & $\mathrm{Y}$ \\
16 & $\mathrm{~F}$ & 59 & Shoulder & $\mathrm{Y}$ \\
17 & $\mathrm{M}$ & 49 & Groin & $\mathrm{N}$ \\
18 & $\mathrm{M}$ & 34 & Jugular & $\mathrm{N}$ \\
19 & $\mathrm{~F}$ & 90 & Groin & $\mathrm{N}$ \\
20 & $\mathrm{M}$ & 60 & Neck & $\mathrm{N}$ \\
21 & $\mathrm{~F}$ & 34 & Parotid & $\mathrm{N}$ \\
22 & $\mathrm{M}$ & 67 & Neck & $\mathrm{Y}$ \\
23 & $\mathrm{M}$ & 28 & Axillary & \\
\hline
\end{tabular}

Nodal melanocytic nevi

\begin{tabular}{|c|c|c|c|c|c|c|}
\hline No. & Sex & Age & Location & Sentinel & Prior MM & Primary tumor \\
\hline 1 & $\mathrm{~F}$ & 72 & Axilla & $\mathrm{N}$ & - & Breast ca \\
\hline 2 & $\mathrm{~F}$ & 59 & Axilla & $\mathrm{N}$ & - & Breast ca \\
\hline 3 & $\mathrm{M}$ & 64 & Pelvic & $\mathrm{N}$ & - & Prostate ca \\
\hline 4 & $\mathrm{~F}$ & 73 & Breast & $\mathrm{Y}$ & - & Breast ca \\
\hline 5 & $\mathrm{~F}$ & 37 & Axilla & $\mathrm{N}$ & - & Breast ca \\
\hline 6 & $\mathrm{~F}$ & 46 & Axilla & $\mathrm{N}$ & - & Breast ca \\
\hline 7 & $\mathrm{~F}$ & 60 & Axilla & $\mathrm{N}$ & - & Breast ca \\
\hline 8 & $\mathrm{~F}$ & 53 & Pelvic & $\mathrm{N}$ & - & Leiomyosarcoma \\
\hline 9 & $\mathrm{M}$ & 62 & Jugular & $\mathrm{N}$ & - & Unknown Primary \\
\hline 10 & $\mathrm{~F}$ & 36 & Breast & $\mathrm{Y}$ & - & Breast ca \\
\hline 11 & $\mathrm{~F}$ & 40 & Groin & $\mathrm{Y}$ & + & \\
\hline 12 & $\mathrm{M}$ & 58 & Groin & $\mathrm{Y}$ & + & \\
\hline 13 & $\mathrm{M}$ & 40 & Neck & $\mathrm{Y}$ & + & \\
\hline 14 & $\mathrm{~F}$ & 30 & Axilla & $\mathrm{Y}$ & + & \\
\hline 15 & $\mathrm{~F}$ & 49 & Axilla & $\mathrm{Y}$ & + & \\
\hline 16 & $\mathrm{~F}$ & 59 & Axilla & $\mathrm{N}$ & - & Breast ca \\
\hline 17 & $\mathrm{~F}$ & 50 & Breast & $\mathrm{Y}$ & - & Breast ca \\
\hline \multicolumn{7}{|c|}{ Subcapsular rest } \\
\hline 1 & $\mathrm{M}$ & 63 & Inguinal & Y & + & \\
\hline
\end{tabular}

Immunoreactivity for cytoplasmic nestin was detected using the Ultra View Universal Alkaline Phosphatase Red Detection Kit to avoid difficulty in interpreting nestin staining in cells with pigmented cytoplasm. Appropriate positive and negative controls were included for each run of immunostaining. Nestin is expressed in vascular endothelial cells, so lymph node blood vessels served as internal positive controls. Primary antibodies were omitted as negative controls. The expression of nestin antibodies was qualitatively scored as 0 (no staining), $1+$ (weak), $2+$ (moderate) and $3+$ (strong).

Immunostaining for goat anti-SOX2 antibodies was performed manually. Briefly, the unstained slides were blocked using 1\% BSA (bovine serum antigen) and subsequently incubated with the primary antibody (Neuromics, 1:200, no. GT15098) for $35 \mathrm{~min}$. Secondary antibody (rabbit/mouse/goat, DAKO; no. K0679) was applied and incubated for 30 min, and $\mathrm{DAB}+$ chromogen was incubated for 2 min according to the manufacture's protocol. Between each step, Tris-buffer $(0.5 \%$ Tween $20, \mathrm{pH}$ 7.4) was used for washing. Faint, blush-like cytoplasmic SOX2 was detected in lymph node germinal centers and was used as internal positive control. Primary antibodies were omitted as negative controls. For nodal melanocytic nevi, SOX2 immunostaining was applied to 16 cases, as the melanocytic focus of interest was lost in one case upon serial sectioning of the block.

\section{Statistics}

The statistical association between each antibody and the melanocytic lesions was analyzed using

Table 2 Nestin and SOX2 immunostaining of metastatic melanomas

\begin{tabular}{lcc}
\hline No. & Nestin & SOX2 \\
\hline 1 & $3+$ & + \\
2 & $3+$ & - \\
3 & Rare $3+$ & - \\
4 & $3+$ & + \\
5 & Rare $3+$ & - \\
6 & Rare $3+$ & - \\
7 & $3+$ & + \\
8 & $3+$ & + \\
9 & $3+$ & + \\
10 & $3+$ & - \\
11 & $3+$ & - \\
12 & $3+$ & - \\
13 & $3+$ & - \\
14 & Diffuse $1+$ & - \\
15 & Rare $3+$ & + \\
16 & $3+$ & - \\
17 & $3+$ & + \\
18 & $3+$ & + \\
19 & $3+$ & + \\
20 & $3+$ & - \\
21 & $3+$ & + \\
22 & $3+$ & + \\
23 & $3+$ & \\
\hline
\end{tabular}



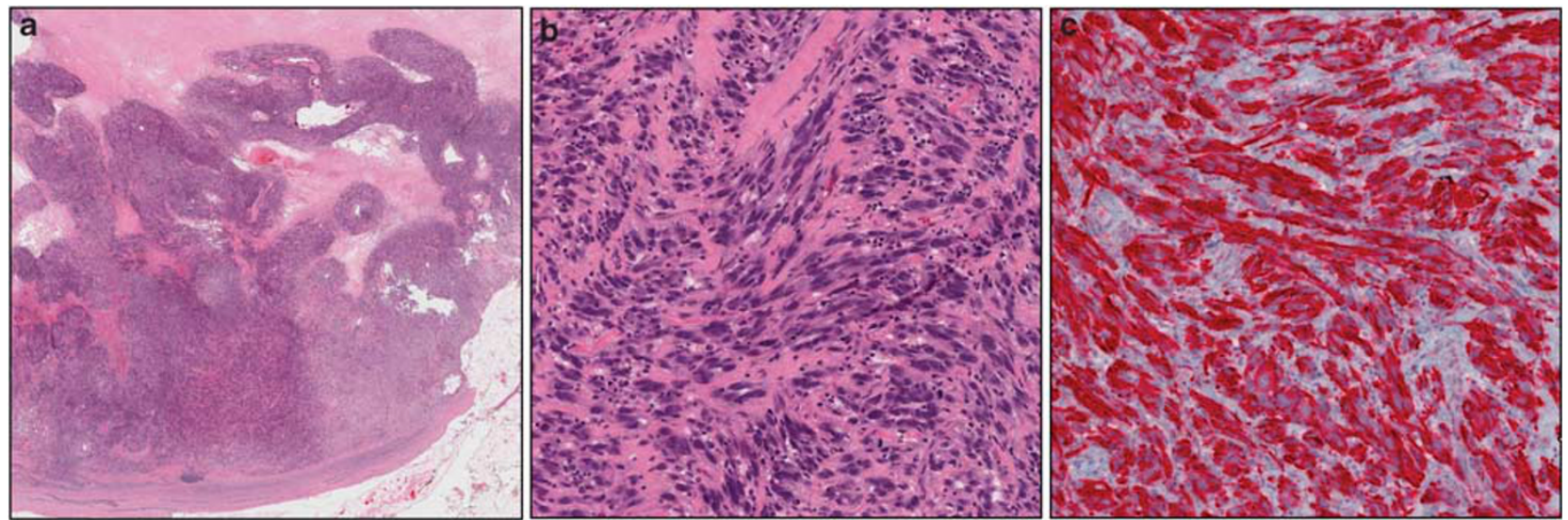

Figure 1 (a) Metastatic melanoma (MetM) in lymph node, hematoxylin and eosin (H\&E; $\times 6.7$ magnification). (b) H\&E $(\times 100$ magnification). (c) Nestin strongly $(3+)$ and diffusely highlights MetM ( $\times 100$ magnification).
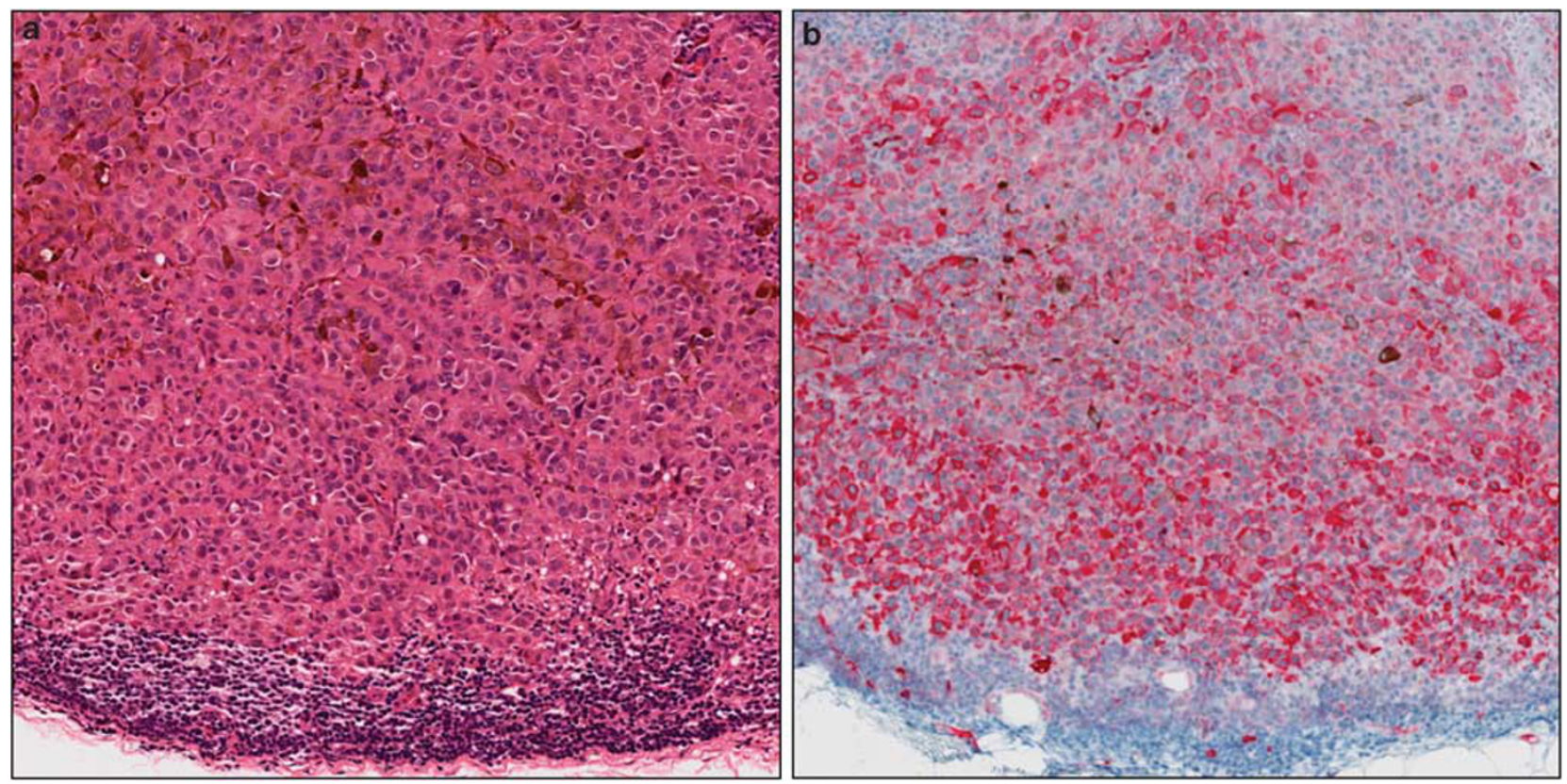

Figure 2 (a) Pigment-producing epithelioid melanoma cells, hematoxylin and eosin ( $\times 100$ magnification). (b) Nestin expression is decreased in the pigment-producing tumor cells compared to the amelanotic tumor cells ( $\times 100$ magnification).

Fisher's exact test. All reported $P$-values were based on a two-sided hypothesis, and a $P$-value $<0.05$ was considered statistically significant.

\section{Results}

\section{Nestin Expression in Lymph Nodes with Metastatic Melanoma}

All 23 cases of metastatic melanoma harbored intraparenchymal tumor cells that were epithelioid and/ or spindled in morphology and showed cytological features of malignancy (marked atypia, mitoses, etc). Some tumor cells contained melanin pigment, whereas some were amelanotic.
Immunostain for nestin revealed strong $(3+)$ and diffuse cytoplasmic positivity in 18 lymph nodes ( $n=18 / 23,78 \%$; Figure 1a-c). Expression of nestin in rare cells with strong $(3+)$ cytoplasmic positivity was seen in 4 lymph nodes $(n=4 / 23,17 \%)$, whereas 1 lymph node showed diffuse, weak $(1+)$ positivity $(n=1 / 23,4 \%)$. If all tumor cells with strong $(3+)$ nestin immunostain were considered positive, the sensitivity of nestin in detecting metastatic melanoma was 96\% (Table 2). Interestingly, nestin was consistently expressed in metastatic melanomas with spindle cell morphology, highlighting $>85 \%$ of these tumors' cells (Figure 1a-c). In metastatic melanomas with epithelioid morphology, the melanin-producing tumor cells showed decreased 

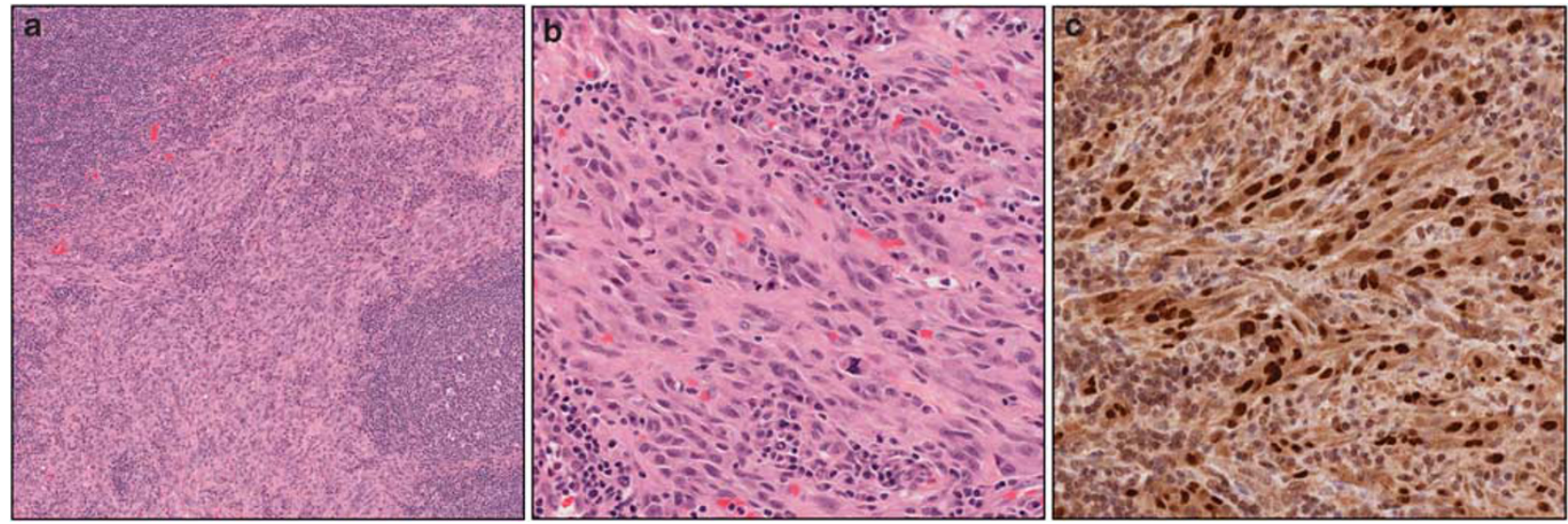

Figure 3 (a) Metastatic melanoma $($ MetM) in lymph node, hematoxylin and eosin $(\mathrm{H} \& \mathrm{E} ; \times 50$ magnification). (b) H\&E $(\times 200$ magnification). (c) MetM is positive for nuclear SOX2 $(\times 200$ magnification).
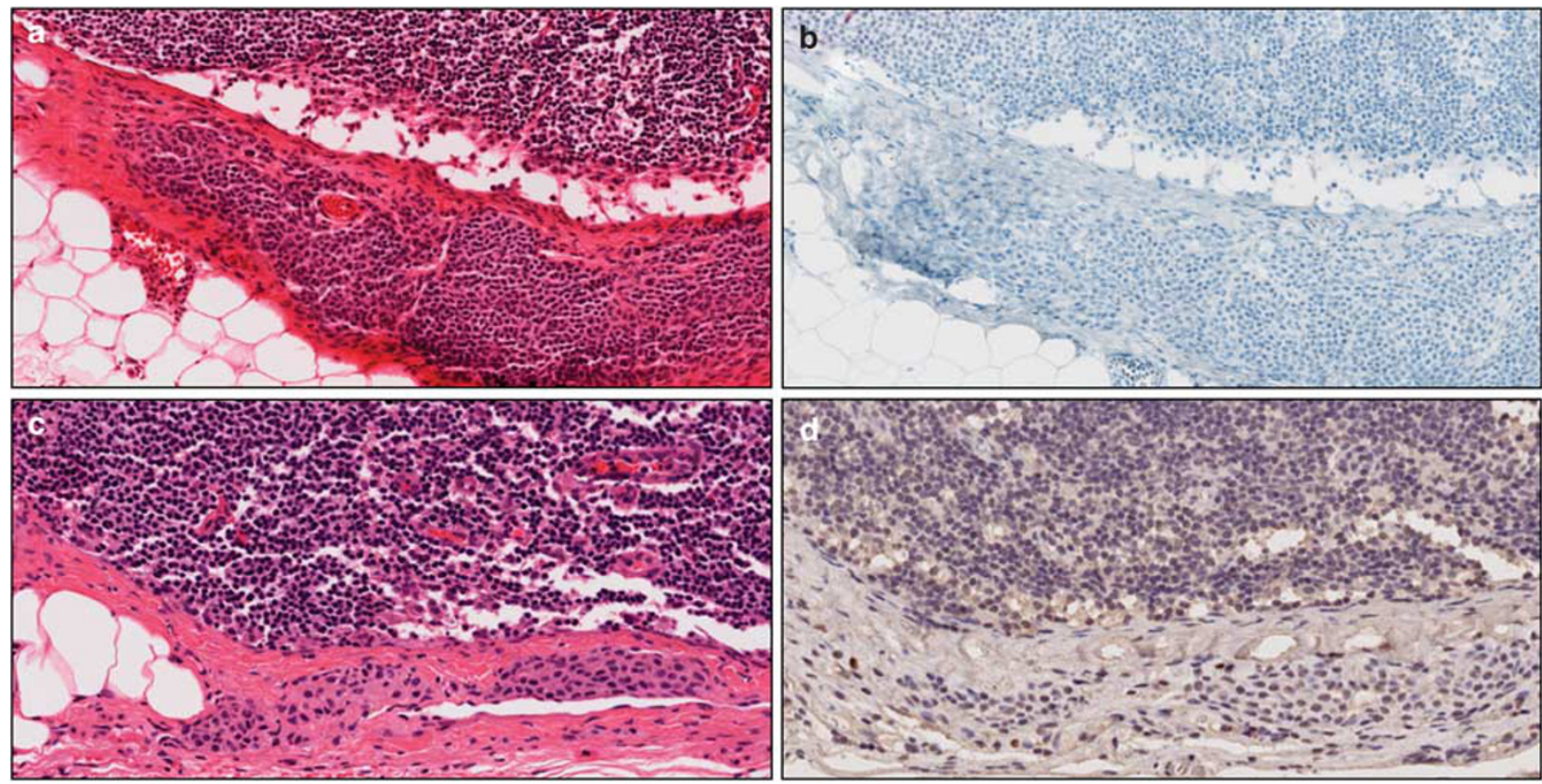

Figure 4 (a) Nodal melanocytic nevus $(\mathrm{nMN})$, hematoxylin and eosin $(\mathrm{H} \& \mathrm{E} ; \times 100$ magnification). (b) Nestin is negative in $\mathrm{nMN}(\times 100$ magnification). (c) Another nMN, H\&E ( $\times 100$ magnification). (d) SOX2 is negative in nMN ( $\times 100$ magnification).

nestin staining (Figure 2a-b) compared with the amelanotic tumor cells. However, within the melanotic tumor foci, scattered tumor cells with strong $(3+)$ nestin staining were readily identifiable. Overall, nestin immunostain was highly sensitive for metastatic melanomas, especially in spindle and amelanotic tumor cells.

\section{SOX2 Expression in Lymph Nodes with Metastatic Melanoma}

Nuclear SOX2 staining was positive in 13 of 23 metastatic melanomas $(n=13 / 23,57 \%$; Figure 3a-c; Table 2). However, no significant difference in SOX2 staining was observed between the spindle and epithelioid tumor cells (both melanotic and amelanotic). Overall, the sensitivity of SOX2 in detecting metastatic melanoma in lymph nodes was somewhat lower compared with nestin.

\section{Nestin Expression in Nodal Melanocytic Nevi}

For nodal melanocytic nevi $(n=17)$, the melanocytic foci were either capsular or trabecular in location. Fifteen nodal nevi showed no nestin expression $(n=15 / 17,88 \%)$ and 2 contained rare cells with weak $(1+)$ /blush-like positivity $(n=2 / 17$, $12 \%$; Figure $4 \mathrm{a}-\mathrm{b}$ and Table 3 ). One of these two lymph nodes was from a patient with no history of melanoma, and the other was from a patient with a 
history of melanoma. Overall, nestin staining was negative in the majority of nodal melanocytic nevi $(88 \%)$ and strongly positive in metastatic melanomas $(96 \% ; P<0.001)$.

\section{SOX2 Expression in Nodal Melanocytic Nevi}

Nuclear SOX2 expression was negative in 13 nodal nevi ( $n=13 / 16,81 \%$ ) (Figure $4 c-d$ ) and positive in 3 nodal nevi $(n=3 / 16,19 \%$; Table 3$)$. The three cases that were positive for SOX2 were not the same nodal nevi that demonstrated weak $1+$ nestin staining. Overall, SOX2 was negative in the majority of nodal nevi (81\%) and positive in $57 \%$ of metastatic melanomas $(P=0.02)$.

Table 3 Nestin and SOX2 immunostaining of nodal melanocytic nevi

\begin{tabular}{lcc}
\hline No. & Nestin & SOX2 \\
\hline 1 & - & - \\
2 & - & - \\
3 & - & $1+$ \\
4 & - & Focus gone \\
5 & - & - \\
6 & - & $1+$ \\
7 & Few $1+$ & - \\
8 & - & $1+$ \\
9 & - & - \\
10 & - & - \\
11 & - & - \\
12 & - & - \\
13 & - & - \\
14 & Rare cells $1+$ & - \\
15 & - & - \\
16 & - & - \\
17 & - & - \\
Subcapsular rest & Rare cells $3+$ & \\
1 & & \\
\hline
\end{tabular}

\section{Nestin and SOX2 Expression in Nodal 'Subcapsular/ Intranodal Melanocytic Rest'}

One case that was originally diagnosed as 'subcapsular/intranodal melanocytic rest' was included in this study. The patient had a history of malignant melanoma, but histological sections of the primary tumor could not be obtained for comparison at the time of lymph node evaluation. The melanocytes within the subcapsular/intranodal melanocytic rest, some of which were pigmented, appeared bland and had smooth nuclear contours and inconspicuous nucleoli. A diagnosis of nevus aggregate was thus favored (Figure 5a).

Immunostain for nestin showed subsets of cells with strong $(3+)$ nestin positivity in this melanocytic aggregate (Figure 5b). Compared with what we observed in other nodal melanocytic nevi, strong nestin expression was unusual for terminally differentiated nevocytes. Careful inspection of the nuclei showed no SOX2 expression (Figure 5c). Upon reexamining the original H\&E-stained sections, the melanocytes appeared larger than what would be expected of a typical nevus, and the nuclei were also larger. Review of the patient's electronic medical record revealed metastatic melanoma to the same lymph node region 2 years later. Retrospectively, this lymph node likely harbored metastatic melanoma rather than a nodal melanocytic nevus.

\section{Metastatic Desmoplastic Melanoma to the Lymph Node}

Desmoplastic melanoma is a rare variant of malignant melanoma that is typically negative, or at best focally positive, for melan-A and HMB-45. ${ }^{12}$ As such, S100 protein is one of the most commonly applied marker for this tumor. A rare case of metastatic, melan-A-negative desmoplastic melanoma was identified in our database and included in this study (Figure 6a-b and e). The lymph node showed nests of spindled tumor cells infiltrating the nodal parenchyma from the periphery of the lymph node.
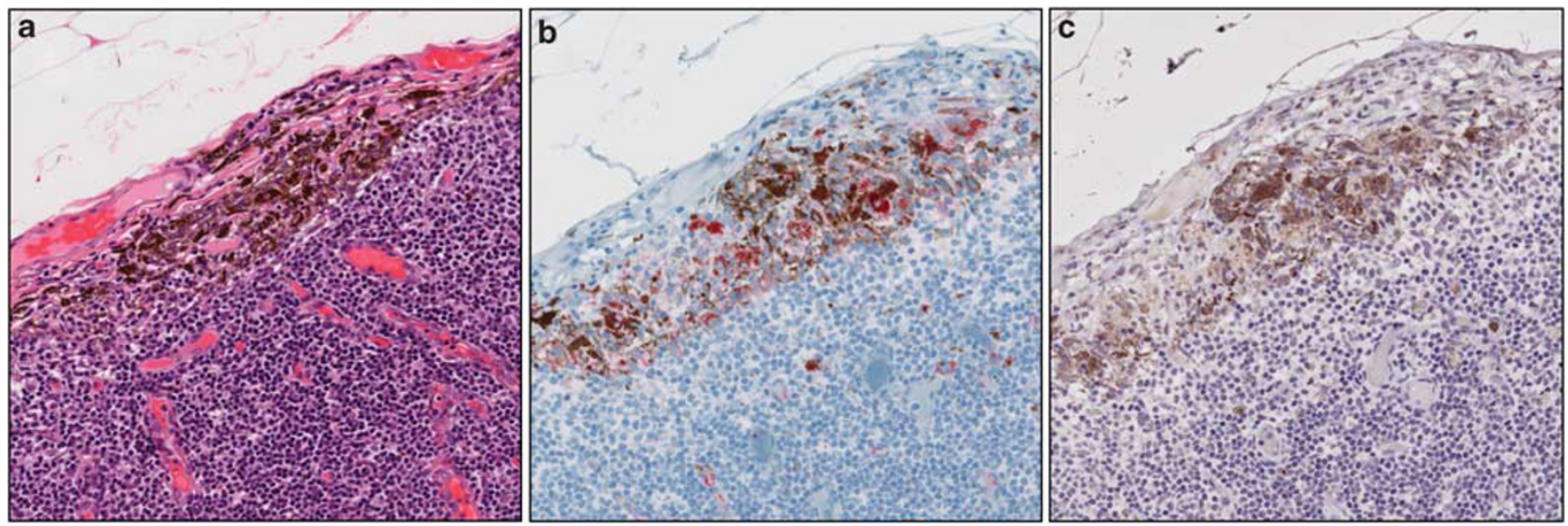

Figure 5 (a) 'Subcapsular/intranodal melanocytic rest' with bland cytology, hematoxylin and eosin ( $\times 141$ magnification). (b) Subsets of cells with strong $(3+)$ Nestin positivity $(\times 141$ magnification). (c) SOX2 is negative $(\times 141$ magnification). 

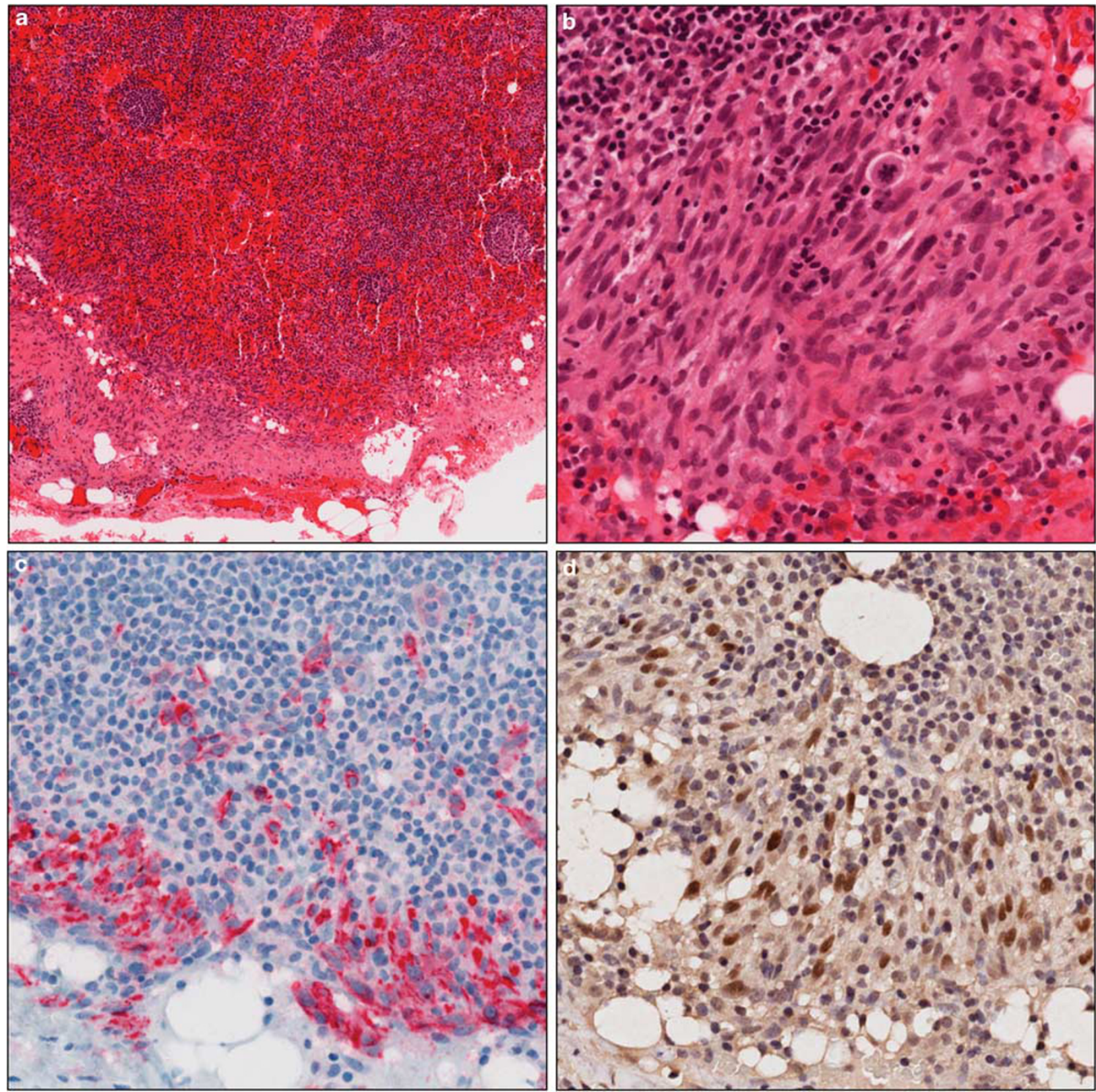

Figure 6 (a) Metastatic desmoplastic melanoma infiltrating from the periphery of the lymph node, hematoxylin and eosin $(\mathrm{H} \& \mathrm{E} ; \times 40$ magnification). (b) H\&E ( $\times 200$ magnification). (c) Nestin strongly $(3+)$ highlighted the tumor cells $(\times 200$ magnification), and (d) SOX2 nuclear staining was also positive $(\times 200$ magnification). (e) Melan-A is negative in the metastatic melanoma cells. (f) S100 protein highlights both desmoplastic melanoma and follicular dendritic cells ( $\times 200$ magnification).

Nestin strongly highlighted the tumor cells (Figure 6c), and SOX2 nuclear staining was also positive (Figure 6d). Both markers highlighted the tumor cells with high sensitivity, but not the background follicular dendritic cells, as observed with S100 protein immunostain (Figure 6f).

\section{Discussion}

One of the diagnostic pitfalls in melanoma sentinel lymph node evaluation is the presence of nodal melanocytic nevi. ${ }^{8}$ Stewart and Copeland ${ }^{28}$ initially described these intranodal nevocellular aggregates in 1931, yet the mechanism by which these nevus cells reach and reside within the lymph nodes remains poorly understood. Two theories have been proposed to explain the origin of nodal melanocytic nevi. ${ }^{29}$ One theory invokes the hypothesis that nodal melanocytic nevi arise from aberrant or arrested migration of neural crest cells during embryogenesis, whereas the opposing theory, or the so-called 'benign metastasis theory' attributes nodal nevi to the mechanical transport of nevus 

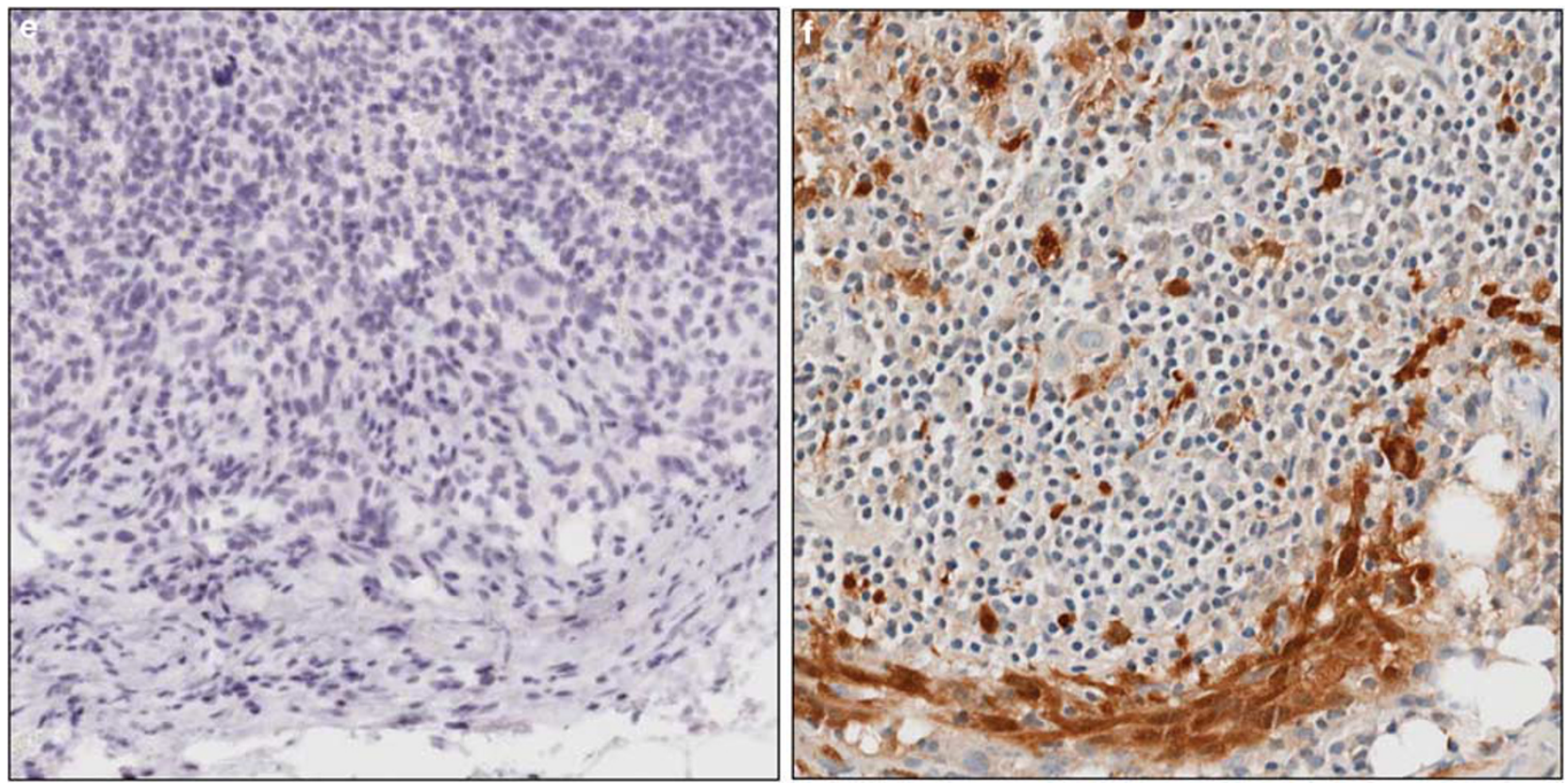

Figure 6 Continued.

cells from cutaneous melanocytic nevi to lymph nodes via lymphatic drainage.

Even though most investigators consider these nevocellular aggregates benign because of their bland cytology and lack of malignant behavior, the presence of these melanocytes, especially in patients with a history of melanoma, can make the evaluation of sentinel lymph node biopsies challenging for practicing pathologists. ${ }^{29}$ Unfortunately, immunohistochemical markers such as melan-A/ MART1, S100 protein and SOX10 demonstrated limited utility in differentiating metastatic melanomas from melanocytic nevi in lymph nodes. ${ }^{13,14,16}$

In the present study, we showed that the neural stem/progenitor cell markers nestin and SOX2 exhibited high specificity in differentiating metastatic melanomas from nodal melanocytic nevi. In addition, nestin immunostain showed superior sensitivity compared with SOX2 and exhibited preferential staining in melanomas with spindle cell morphology and amelanotic tumor cells. As nestin is a stem/neural progenitor cell marker, the decreased expression of nestin in melanin-producing tumor cells may be related to the more 'differentiated' state of these cells.

When assessing benign nodal nevi and metastatic melanomas, the combined use of nestin and SOX2 immunostains further enhanced the sensitivity and specificity of these two markers in detecting and differentiating melanocytic lesions. In this study, all cases of nodal metastatic melanomas showed either strong nestin (diffusely or in rare cells) or SOX2 positivity, but no nodal melanocytic nevi were positive for both markers. Our experience also indicates that when encountering histologically ambiguous melanocytic lesions, a strong $(3+)$ nestin staining alone is highly suggestive of metastatic melanoma rather than nodal melanocytic nevi.

We specifically selected metastatic melanomas and nodal melanocytic nevi with indisputable histomorphology for this study. In reality, diagnostic challenge often arises when single or rare melanocytes are detected by immunohistochemistry, and in these situations, the classification of such lesions as benign or malignant is not so clear cut. Our study included one case of 'subcapsular/intranodal melanocytic rest' that showed deceptively bland cytology and a benign diagnosis was favored originally. Correlation with strong cytoplasmic nestin staining and clinical follow-up suggested that the 'melanocytic rest' was more likely a histologically ambiguous melanoma micrometastasis rather than a benign melanocytic nevus. We propose that the diagnostic accuracy of sentinel lymph node evaluation, especially when confronted with melanocytes of non-classic histological features, could potentially benefit from nestin and SOX2 immunostains. Future studies involving a larger number of lymph node specimens are needed to address the value of nestin and SOX2 in borderline melanocytic lesions of uncertain malignant potential.

A limiting factor in this study was tissue loss, specifically the loss of melanocytic nevi, upon serial sectioning of tissue blocks. At the outset of this study, six additional cases of nodal melanocytic nevi were identified through a search of our institutional database. However, because of tissue exhaustion, those cases were excluded from this study. One case of a nodal melanocytic nevus had adequate tissue for only one immunostain (nestin) and was thus included in this study. When small nodal melanocytic lesions are encountered in the 
future, it may be advantageous that additional tissue sections be preserved in the event that further immunohistochemical analysis may be needed for the interpretation. A cocktail of anti-mouse nestin (cytoplasmic)/anti-goat SOX2 (nuclear) antibodies can also be considered for future studies to preserve tissue.

Lastly, one case of metastatic desmoplastic melanoma merits special mention. Unlike conventional melanomas, desmoplastic melanomas are typically positive for S100 protein and SOX10, but are negative, or at best focally positive for melan-A and HMB-45. ${ }^{12,13}$ When pathologists encounter desmoplastic melanoma lymph node biopsies, S100 protein immunostain, with all of its limits, and SOX10 are the best options. Here we demonstrated that both nestin and SOX2 immunostains strongly highlighted nodal metastatic desmoplastic melanoma, whereas S100 protein was positive in both the tumor cells and the follicular dendritic cells. We believe nestin and SOX2 may be superior to S100 when evaluating desmoplastic melanomas in sentinel lymph nodes.

In summary, we have provided evidence that the neural stem/progenitor cell markers nestin and SOX2 can effectively differentiate metastatic melanomas from benign nodal melanocytic nevi and potentially serve as powerful diagnostic adjuncts in melanoma sentinel lymph node biopsies and staging.

\section{Acknowledgements}

We thank Walter Clement and Stacey Yates for imaging and technical support, and Neha Dahiya for processing immunohistochemistry orders at the core research lab.

\section{Disclosure/conflict of interest}

The authors declare no conflict of interest.

\section{References}

1 Gershenwald JE, Ross MI. Sentinel-lymph-node biopsy for cutaneous melanoma. N Engl J Med 2011;364: 1738-1745.

2 Morton DL, Thompson JF, Cochran AJ, et al. Sentinelnode biopsy or nodal observation in melanoma. N Engl J Med 2006;355:1307-1317.

3 Gershenwald JE, Andtbacka RH, Prieto VG, et al. Microscopic tumor burden in sentinel lymph nodes predicts synchronous nonsentinel lymph node involvement in patients with melanoma. J Clin Oncol 2008; 26:4296-4303.

4 Stebbins WG, Garibyan L, Sober AJ. Sentinel lymph node biopsy and melanoma: 2010 update Part II. J Am Acad Dermatol 2010;62:737-748; quiz 749-750.

5 Balch CM, Gershenwald JE, Atkins MB, et al. Melanoma of the skin. In: Edge SB, Byrd DR, Compton CC, et al. (eds), AJCC Cancer Staging Manual, 7th edn. Springer: New York; 2009, pp 325-344.

6 Scheri RP, Essner R, Turner RR, et al. Isolated tumor cells in the sentinel node affect long-term prognosis of patients with melanoma. Ann Surg Oncol 2007; 14:2861-2866.

7 Biddle DA, Evans HL, Kemp BL, et al. Intraparenchymal nevus cell aggregates in lymph nodes: a possible diagnostic pitfall with malignant melanoma and carcinoma. Am J Surg Pathol 2003;27:673-681.

8 Carson KF, Wen DR, Li PX, et al. Nodal nevi and cutaneous melanomas. Am J Surg Pathol 1996;20: 834-840.

9 Murray CA, Leong WL, McCready DR, et al. Histopathological patterns of melanoma metastases in sentinel lymph nodes. J Clin Pathol 2004;57:64-67.

10 Howell BG, Lipa JE, Ghazarian DM. Intracapsular melanoma: a new pitfall for sentinel lymph node biopsy. J Clin Pathol 2006;59:891-892.

11 Shidham VB, Qi DY, Acker S, et al. Evaluation of micrometastases in sentinel lymph nodes of cutaneous melanoma: higher diagnostic accuracy with Melan-A and MART-1 compared with S-100 protein and HMB45. Am J Surg Pathol 2001;25:1039-1046.

12 Ohsie SJ, Sarantopoulos GP, Cochran AJ, et al. Immunohistochemical characteristics of melanoma. J Cutan Pathol 2008;35:433-444.

13 Nonaka D, Chiriboga L, Rubin BP. Sox10: a panschwannian and melanocytic marker. Am J Surg Pathol 2008;32:1291-1298.

14 Lohmann CM, Iversen K, Jungbluth AA, et al. Expression of melanocyte differentiation antigens and ki-67 in nodal nevi and comparison of ki-67 expression with metastatic melanoma. Am J Surg Pathol 2002;26: 1351-1357.

15 Mahmood MN, Lee MW, Linden MD, et al. Diagnostic value of HMB-45 and anti-melan A staining of sentinel lymph nodes with isolated positive cells. Mod Pathol 2002;15:1288-1293.

16 Blochin E, Nonaka D. Diagnostic value of Sox10 immunohistochemical staining for the detection of metastatic melanoma in sentinel lymph nodes. Histopathology 2009;55:626-628.

17 Boiko AD, Razorenova OV, van de Rijn M, et al. Human melanoma-initiating cells express neural crest nerve growth factor receptor CD271. Nature 2010;466: 133-137.

18 Lendahl U, Zimmerman LB, McKay RD. CNS stem cells express a new class of intermediate filament protein. Cell 1990;60:585-595.

19 Florenes VA, Holm R, Myklebost O, et al. Expression of the neuroectodermal intermediate filament nestin in human melanomas. Cancer Res 1994;54: 354-356.

20 Brychtova S, Fiuraskova M, Hlobilkova A, et al. Nestin expression in cutaneous melanomas and melanocytic nevi. J Cutan Pathol 2007;34:370-375.

21 Klein WM, Wu BP, Zhao S, et al. Increased expression of stem cell markers in malignant melanoma. Mod Pathol 2007;20:102-107.

22 Bakos RM, Maier T, Besch R, et al. Nestin and SOX9 and SOX10 transcription factors are coexpressed in melanoma. Exp Dermatol 2010;19:e89-e94.

23 Laga AC, Zhan Q, Weishaupt C, et al. SOX2 and nestin expression in human melanoma: an immunohistochemical and experimental study. Exp Dermatol 2011;20:339-345. 
24 Piras F, Perra MT, Murtas D, et al. The stem cell marker nestin predicts poor prognosis in human melanoma. Oncol Rep 2010;23:17-24.

25 Fusi A, Reichelt U, Busse A, et al. Expression of the stem cell markers nestin and CD133 on circulating melanoma cells. J Invest Dermatol 2011;131: 487-494.

26 Tanaka S, Kamachi Y, Tanouchi A, et al. Interplay of SOX and POU factors in regulation of the Nestin gene in neural primordial cells. Mol Cell Biol 2004;24: 8834-8846.

27 Takahashi K, Yamanaka S. Induction of pluripotent stem cells from mouse embryonic and adult fibroblast cultures by defined factors. Cell 2006;126:663-676.

28 Stewart FW, Copeland MM. Neurogenic sarcoma. Am J Cancer 1931;15:1235.

29 Patterson JW. Nevus cell aggregates in lymph nodes. Am J Clin Pathol 2004;121:13-15. 